\title{
Quelques Elements de Bilan des Recherches Sur La Mobilité Residentielle en France
}

\author{
Catherine Bonvalet * \\ Jacques Brun ${ }^{* *}$
}

\begin{abstract}
$\mathrm{R}$ permite conhecer como é que os indivíduos se adaptam às diferentes mudanças na sociedade contemporânea, seja no que diz respeito à actividade económica, à vida privada, ao progresso técnico, ou ainda aos modos de urbanização. $O$ estudo da mobilidade residencial, que não pode ser dissociado de outras formas de mobilidade, como a mobilidade quotidiana, revela-se ser um bom indicador das profundas transformações, sociais, demográficas, políticas, que atravessam o mundo urbano.

O tema da mobilidade foi objecto de numerosos estudos e pesquisas por parte de demógrafos, economistas, geógrafos, historiadores, estatísticos, sociólogos. Mas, mesmo tendo estes trabalhos permitido um progresso considerável no conhecimento da mobilidade, os problemas subsistem, na compreensão dos mecanismos e na análise e medição da própria mobilidade.

Palavras-chave : mobilidade geográfica; migração e mobilidade espacial; evolução das estruturas demográficas e familiares; análise transversal e longitudinal da mobilidade.
\end{abstract}

\section{La Mobilité: mesures et évolutions}

\section{La Mesure de la Mobilité}

Les termes de mobilité géographique, migration et mobilité spatiale sont souvent employés indifféremment pour désigner un changement durable de lieu. Pourtant des différences existent dans leur définition et leur utilisation. Le concept de migration désigne souvent de manière plus ou moins explicite des déplacements internationaux ou interrégionaux; le concept de mobilité géographique comprend généralement ces mouvements, mais également les mouvements à l'intérieur d'une agglomération. Mais ces deux concepts ne rendent pas compte des mouvements pendulaires ou "migrations alternantes", ni des divers autres types de déplacements liés aux affaires, aux loisirs, au commerce ou aux vacances. La mobilité résidentielle ne constitue donc qu'un type particulier de la mobilité spatiale, à côté de la mobilité quotidienne, des migrations touristiques, des voyages d'affaires...

Pour appréhender la mobilité avec précision, deux dimensions doivent être prises en compte: l'espace et la durée. En démographie, le concept de migration est défini comme "un ensemble de déplacements ayant pour effet de transférer la résidence des intéressés d'un certain lieu d'origine ou lieu de départ à un certain lieu de destination ou lieu d'arrivée, la résidence étant le lieu où les individus ont coutume d'habiter" (Henry, 1981). C'est donc à partir d'un changement de logement au cours d'une certaine période que la mobilité résidentielle peut être appréhendée ${ }^{\perp}$.

En France, plusieurs sources permettent de mesurer la mobilité :

- Le recensement qui constitue la source essentielle des données sur la mobilité. Grâce à une question sur le lieu de résidence l'année du

\footnotetext{
* Demógrafa, Institut National d'Études Démographiques (INED), Directora da Unidade de Pesquisa: Mobilité, territoires, habitat et sociabilité. Contacto: bonvalet@ined.fr

** Professor na Universidade de Paris. Contacto: jbrun@univ-parisl.fr

' En fonction de la distance parcourue lors de ce déménagement, on a l'habitude de distinguer une mobilité locale et une mobilité lointaine.
} 


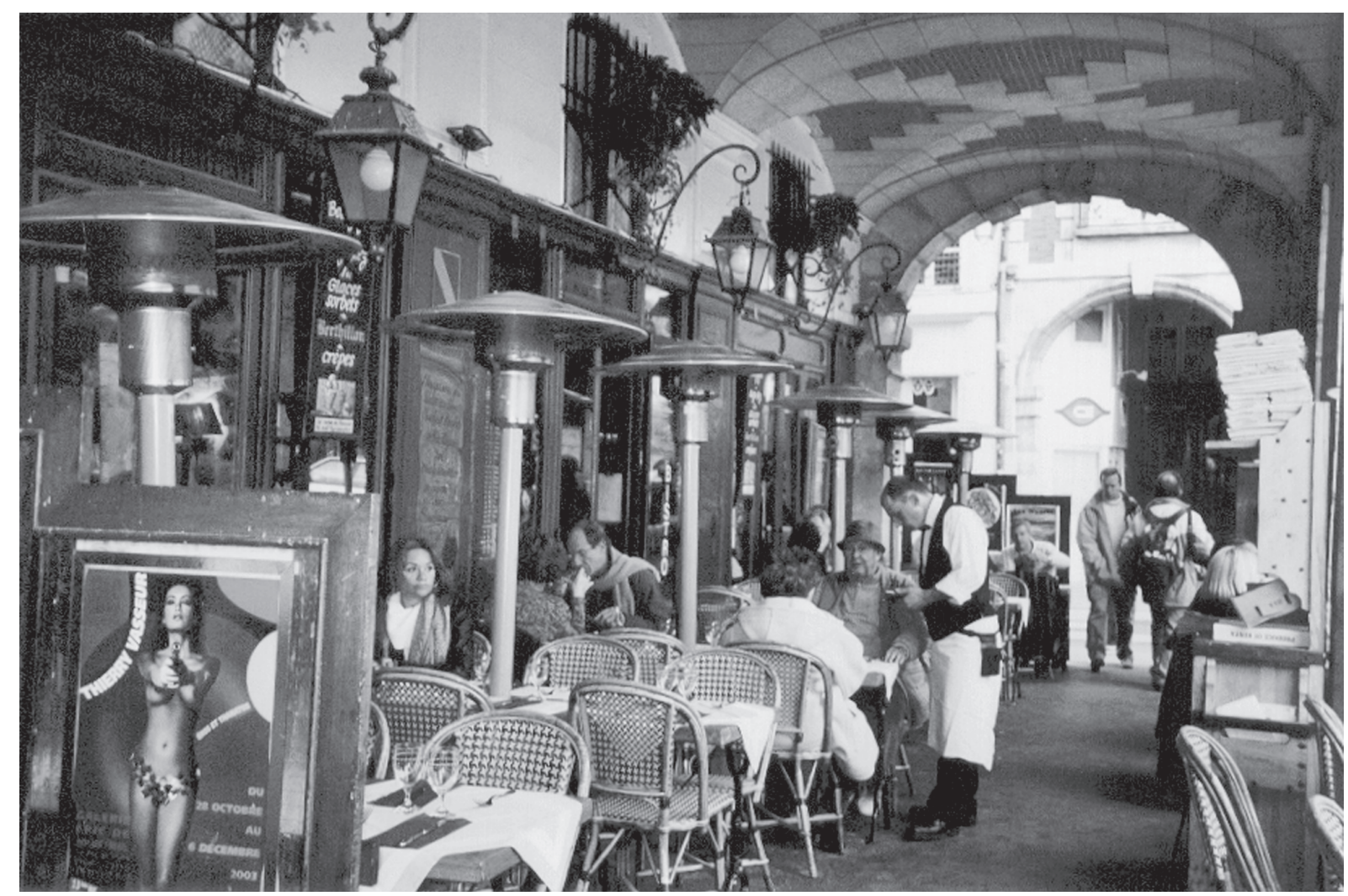

Foto de Vítor Matias Ferreira

précédent recensement, on peut calculer la proportion de personnes ayant changé de logement entre deux recensements, ou, pour éviter l'inconvénient de l'inégalité des intervalles entre deux recensements, des taux annuels de mobilité à différentes échelles. Il est ainsi possible de suivre l'évolution de la mobilité au niveau de la commune, du département, de la région, ou de la France entière.

- Les Enquêtes Logement réalisées tous les quatre ans qui fournissent des données plus complètes sur la mobilité des ménages en fonction de leurs caractéristiques et de celles des logements. On peut ainsi calculer des pourcentages d'emménagés récents parmi les personnes de référence et estimer des taux annuels.

En dehors de ces deux principales sources de données, d'autres enquêtes permettent de suivre les variations de la mobilité dans le temps. Il s'agit en particulier de l'échantillon démographique permanent de l'INSEE (proche d'un échantillon au 1/100) et des Enquêtes Emploi qui interrogent un échantillon de ménages à un an d'intervalle. Il existe aussi des enquêtes spécifiques, sur la base d'échantillons mobiles (enquête EDF) et des enquêtes biographiques. Toutes ces sources, recensements ou enquêtes plus spécifiques, permettent d'analyser les principales caractéristiques des personnes mobiles et immobiles ainsi que d'étudier les flux entre unités administratives ou entre les différents segments du parc de logements. Elles fournissent par ailleurs des informations essentielles sur les raisons des déménagements.

\section{Les Grandes Evolutions de la Mobilité Résidentielle}

Au cours du siècle et demi écoulé, sous l'effet des migrations internes, la France, qui était un pays à 80\% rural, est devenue au trois quarts urbanisée, et, parmi les citadins, trois sur cinq vivent dans une ville de plus de 100000 habitants. Si la croissance de la population urbaine française a été relativement lente pendant tout le XIXe siècle, elle s'est accélérée au cours des quarante dernières années. En 1831, le taux d'urbanisation était de 19\% (contre $44 \%$ en Grande-Bretagne). Il était de 
$36 \%$ en $1891,55 \%$ en 1954 , et a atteint $73 \%$ en 1999. En France, le processus d'urbanisation a donc eu un démarrage tardif et une évolution longtemps modérée. Il a connu un essor plus rapide après la Seconde Guerre Mondiale avec l'afflux des migrants internes et externes vers les villes. De 1954 à 1975, la mobilité résidentielle a crû, prolongeant les tendances observées depuis le milieu du XIXe siècle. Au recensement de 1982, on a pu observer un renversement dans l'évolution séculaire de la mobilité. La période 1975-1982 est en effet marquée par une baisse de la mobilité à toutes les échelles géographiques et à presque tous les âges. Cette baisse de la mobilité s'est prolongée jusqu'au recensement de 1999.

Ces grandes évolutions résultent de la croissance démographique (exode rural, retour des rapatriés en métropole, arrivée à l'âge adulte des enfants du baby-boom) et, jusqu'en 1975, de la croissance de la construction neuve, soutenue par une politique active d'aide à l'accession à la propriété. Ensuite, la moindre pression démographique, la baisse de la construction neuve et les conditions économiques moins favorables ont provoqué une baisse de la mobilité, malgré une politique d'aide à l'accession toujours forte.

Parallèlement à cette baisse de la mobilité, les aires urbaines se sont étendues en absorbant des communes rurales et des petites villes aux alentours. Si les banlieues ont continué à s'étendre et à se densifier, un nouveau phénomène, la périurbanisation, est apparu vers le début des

Taux annuel de mobilité résidentielle entre deux recensements

1968-1975: 9,7\%

1975-1982: $9,4 \%$

1982-1990: $8,6 \%$

1990-1999: $8,1 \%$

Taux pluri-annuel de mobilité résidentielle d'après les Enquêtes Logement

1978-1984: $24,4 \%$

1984-1988: $25,2 \%$

1988-1992: $22,7 \%$

1992-1996: $23,2 \%$ années soixante-dix. Celle-ci s'est prolongée au cours des années quatre-vingt, malgré un mouvement limité de retours vers les pôles urbains.

\section{Les Caractéristiques des Ménages Mobiles}

On connaît bien maintenant les variations de la mobilité au cours du cycle de vie : très forte aux âges jeunes avec un maximum entre 25 et 29 ans, la mobilité chute après 40 ans, toutes distances confondues. On note un très léger relèvement de la mobilité locale pour les personnes de plus de 75 ans, ce qui correspond essentiellement à des départs en institution. La mobilité varie selon la catégorie socio-professionnelle: très faible pour les agriculteurs, faible pour les indépendants, elle est très forte pour les cadres supérieurs. Par ailleurs, elle augmente avec la position hiérarchique des salariés dans l'entreprise. La mobilité à grande distance apparaît comme l'apanage des cadres, alors que les employés et les ouvriers se déplacent plus rarement hors de leur région. Il semble enfin, au vu du recensement de 1990, que les chômeurs aient été plus mobiles que les actifs ayant un emploi.

En résumé, les ménages les plus mobiles sont les jeunes qui décohabitent et cherchent un emploi, puis forment un couple et ont des enfants. A chaque étape du cycle de vie familial et professionnel, les besoins en logement se transforment et les ménages s'efforcent d'adapter leur logement à leur nouvelle situation. Ces changements de résidence se produisent essentiellement pour des raisons liées à l'évolution de la famille, alors que les déménagements à grande distance résultent plus fréquemment de changements professionnels.

Les évolutions qui viennent d'être évoquées présentent des modalités variables selon les agglomérations et les régions. L'exploitation des enquêtes nationales et régionales a donc permis une première accumulation de connaissances de plus en plus précises sur les variations et en particulier les variations conjoncturelles. Néanmoins, il est apparu nécessaire de compléter ces acquis par des travaux spécifiques impliquant soit des analyses secondaires de données de l'INSEE, soit la mise en œuvre d'enquêtes tant quantitatives que qualitatives. 


\section{Vers une Inflexion des Approches Méthodologiques et des Références Théoriques}

Les progrès dans la production et l'exploitation des données censitaires fournissent donc des informations essentielles sur les liens entre les variations de la mobilité résidentielle, dans le temps et dans l'espace, l'évolution des structures familiales et démographiques, et les transformations du marché du logement. Cependant, d'autres catégories de données, sur lesquelles les sources censitaires apportent également des informations, parfois très précises, parfois moins complètes, apparaissent également comme les indicateurs d'autant de "facteurs" entretenant avec la mobilité des interrelations qu'il importe de prendre en compte.

En fait, comme le rappellent les tentatives de définition évoquées plus haut, la mobilité résidentielle est en interaction avec des phénomènes si nombreux et si complexes - en particulier les autres formes de mobilité spatiale, y compris les mouvements des capitaux et de l'information "immatérielle", mais aussi la mobilité sociale - qu'il a fallu compléter les connaissances de base par de nouveaux types de travaux.

Au cours de la deuxième moitié du XXe siècle, les recherches sur la mobilité se sont multipliées dans le cadre de toutes les sciences de la société, et se sont spécialisées au fur et à mesure qu'elles s'approfondissaient. Mais, dans le même temps, des passerelles se sont tissées entre les différentes approches. En particulier, si, au cours des décennies soixante et soixante-dix, les progrès des connaissances ont surtout résulté de travaux effectués à partir de données de l'INSEE, depuis les années quatre-vingt, l'analyse de la mobilité résidentielle s'est développée aussi à partir d'enquêtes retraçant l'histoire migratoire et résidentielle des individus.

Les recherches récentes portent tant sur des données agrégées - produites essentiellement par l'INSEE - que sur des données individuelles recueillies, pour l'essentiel, dans le cadre d'enquêtes spécifiques. La distinction n'a pas perdu sa pertinence, même si l'on note, au fil du temps, une convergence entre ces deux grands types de démarche (Brun, 1993). Elle recoupe en partie la distinction entre deux grandes approches, l'approche transversale et l'approche longitudinale ${ }^{2}$.

Au cours des dernières décennies, une importante évolution a marqué le développement de ces deux approches: au départ les analyses quantitatives reposaient essentiellement sur une appréhension "macro" de la mobilité, par la suite, des analyses micro ont pu être menées à bien. Cette évolution a été favorisée par tout un ensemble de facteurs, que l'on retrouverait dans de nombreux domaines de la recherche en sciences sociales. On peut citer par exemple le développement de nouveaux instruments conceptuels et méthodologiques - telles les analyses longitudinale et biographique - ou encore les progrès de l'informatique... Mais, pour rendre compte de ce "passage du général au particulier", sans doute faudrait-il aussi évoquer d'autres éléments du contexte, comme le reflux d'un certain structuralisme, ou le "retour" de l'acteur individuel sur la scène des sciences sociales...

\section{Analyse Transversale et Analyse Longitudinale de la Mobilité}

\section{Développements de l'analyse transversale et} progrès de la compréhension de la mobilité

Les études et recherches qui relèvent de l'analyse transversale ont connu un important développement au cours des premières décennies après la Seconde Guerre Mondiale. Elles répondent à des besoins urgents de connaissances concernant les processus de peuplement (l'évolution du parc de logements, les migrations internes et externes, le marché du travail et plus globalement l'aménagement du territoire). C'est une période où la question de l'exode rural est encore d'actualité, tandis que celle de l'immigration se pose en termes nouveaux en raison du développement de nouveaux flux migratoires.

Avec l'accélération de l'urbanisation et les modifications du marché du travail (chute des effectifs du secteur primaire, stagnation puis reflux du secteur secondaire, essor du tertiaire), les mouvements se font de moins en moins entre régions et de plus en plus au sein des agglomérations, plus particulièrement des centres vers les banlieues, puis les zones périphériques.

\footnotetext{
${ }^{2}$ L'analyse transversale, appelée encore analyse du moment, est l'étude d'une population, de ses structures et de son comportement dans la synchronie.
} 


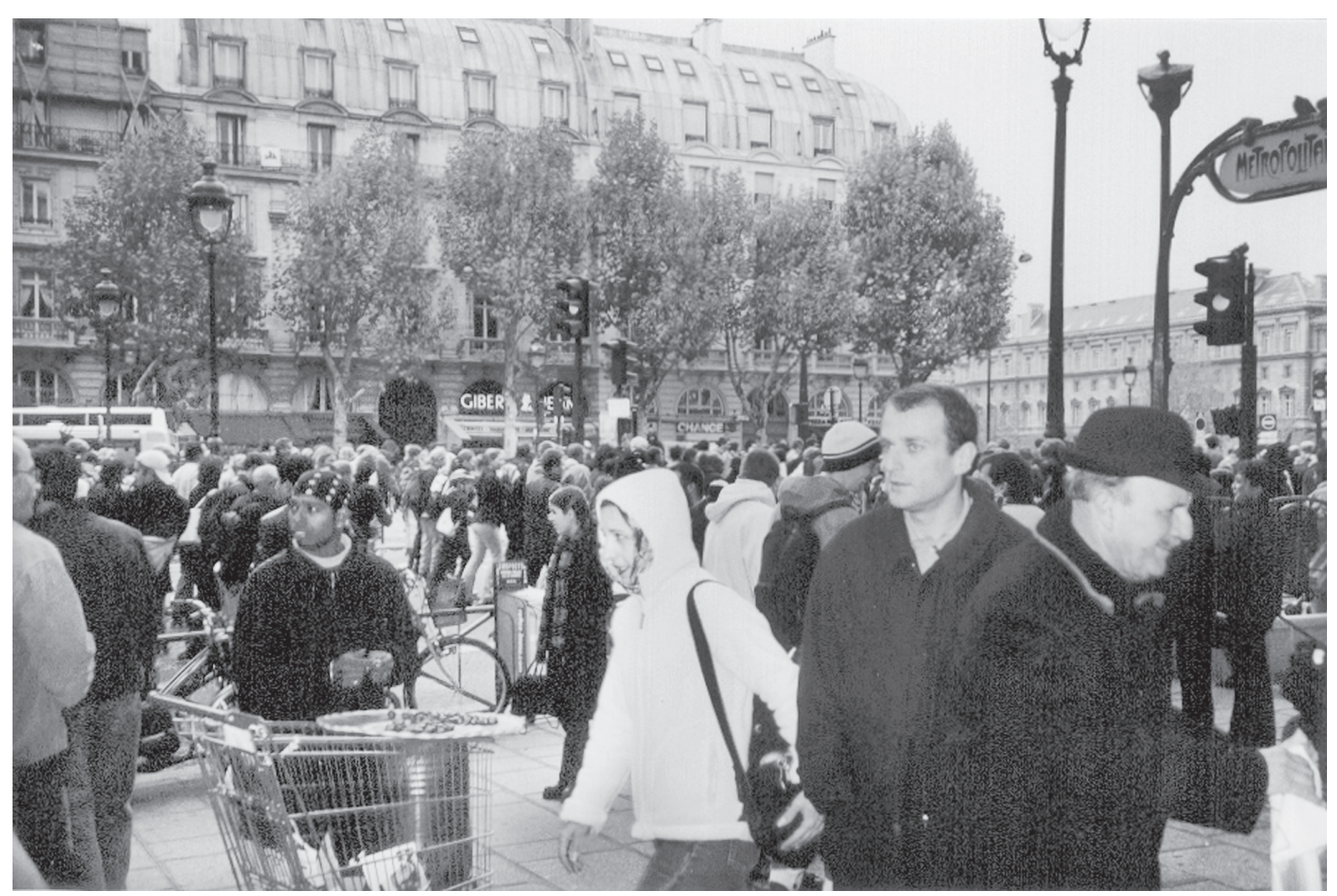

Foto de Vítor Matias Ferreira

Parallèlement à ces changements dans les structures de l'économie et de l'emploi, se modifient aussi les structures du parc (notamment avec l'essor de la maison individuelle dans l'espace périurbain), et celles des ménages (diminution du poids des couples, accroissement de celui des personnes isolées ainsi que des familles monoparentales). L'évolution de la recherche reflète ces changements. On peut ainsi distinguer quatre manières de se saisir des questions de mobilité, qui correspondent - en partie du moins - à des disciplines, mais aussi à des courants de pensée:

1 - Les travaux qui rendent compte des choix de migration, de localisation et de statut d'occupation à l'aide de modèles économétriques; Les logiques qui sous-tendent l'ensemble de ces travaux sont avant tout économiques, même lorsqu'ils sont effectués par des géographes ou des sociologues. Pour rendre compte des comportements de mobilité, les chercheurs font l'hypothèse que l'homme est un être rationnel cherchant à optimiser ses choix. La décision de migrer, d'acheter ou de louer un logement, de résider à tel endroit peut donc être représentée plus ou moins simplement à l'aide d'un modèle économétrique. On trouvera donc des modèles de migration, des modèle de choix du logement (Rosen, 1974) ainsi que des modèles de localisation (Alonso,1964). Dans chaque cas, l'individu examine les avantages et inconvénients des différentes possibilités qui s'offrent à lui et choisit celle qui lui est la plus favorable en termes économiques.

2 - Les travaux qui analysent la mobilité en fonction des événements familiaux et professionnels; La mobilité résidentielle dépend en effet fortement des événements familiaux et de leur fréquence. De nombreuses études ont ainsi traité des différentes étapes du cycle de vie et de leurs incidences sur la mobilité. Une modification de la taille du ménage est une des raisons les plus fréquentes du désir de changer de logement. La nuptialité a fait très tôt l'objet d'analyses de grande précision (Courgeau, 1976). Depuis une vingtaine d'années, les chercheurs se sont intéressés à une étape du cycle de vie familiale de plus en plus importante dans notre société, le divorce. Au total, la multiplication des événements familiaux (mariage, divorce, remise en couple, remariage...) a pour effet une augmen- 
tation sensible de la mobilité résidentielle (Bonvalet, 1985). Les effets des changements familiaux sur la demande de logements ont été modélisés dès le début des années quatre-vingt, surtout dans des travaux anglo-saxons (Ermish, 1981; Holmans, 1983; Dielman, Clark, 1985). Deux autres étapes ont retenu l'attention des chercheurs: la première étape, la décohabitation des jeunes (Bloss, Godard, Galland, Villeneuve-Gokalp), et la retraite (Cribier).

3 - Les travaux qui étudient la mobilité à partir de l'évolution de la structure du parc de logements et de la structure socioprofessionnelle et démographique de ses occupants. Ces travaux ne portent que sur la mobilité locale, considérant que les marchés locaux du logement sont un élément déterminant de la mobilité résidentielle; tout un courant de recherche a mis en évidence l'importance de la structure du parc de logements et des effets de contexte. Ces approches prennent en compte le parc de logements, à l'échelle locale, comme un des éléments déterminants de la mobilité globale à cette échelle. De nombreuses recherches plus récentes traitent de la répartition des classes sociales dans les agglomérations urbaines en fonction du parc de logement (Berger, Brun, Chauviré, Lévy, Rhein). Les relations entre catégories sociales, parc de logements, espace urbain, sont un élément essentiel de toutes les études sur la division sociale de l'espace ou sur la ségrégation. Ces travaux débouchent sur l'analyse de la mobilité, qui n'est pas seulement étudiée à partir des déplacements des hommes, mais aussi à partir des mouvements du parc (Lévy).

Un de leurs éléments novateurs est de permettre de suivre avec plus de précision les processus de peuplement de l'espace urbain et les phénomènes de valorisation ou dévalorisation des différents segments du parc.

4 - Les travaux qui attachent une place essentielle aux liens qui se tissent entre les individus, les familles et les lieux (de l'échelle du quartier à celle de la nation en passant par le pays ou la région). Les sociologues ont apporté un autre éclairage sur les choix opérés par les ménages en termes de statut d'occupation, de type de logement, ou de localisation. L'idée essentielle de ces travaux est que ces choix ne sont pas entièrement soumis aux règles ordinaires du marché et donc à une logique économique selon laquelle chaque ménage serait assigné à une localisation et un statut d'occupation précise, en fonction de sa catégorie socioprofessionnelle, de sa position dans le cycle de vie familial, etc. D'autres logiques, familiales, sociales, affectives, peuvent être évoquées. L'individu ou le ménage ne prendrait donc pas ses décisions uniquement en fonction de ses propres caractéristiques repérées à un moment donné, mais en fonction d'autres critères sociaux - psychologiques, affectifs, comme l'attachement à un lieu -, ou encore en fonction de groupes de référence, qui peuvent être la famille, le groupe social dominant du quartier, la communauté d'origine ${ }^{3}$. Trois types de choix ont fait l'objet d'investigations particulières: le choix entre appartement et maison, le statut d'occupation, la localisation, en particulier le choix d'un quartier.

\section{Approches Longitudinales et Analyses Biographiques}

L'analyse biographique apporte un autre éclairage sur la mobilité en s’intéressant non plus à un individu à un instant donné mais à des générations dont l'histoire est reconstituée. Elle se situe dans le prolongement de l'analyse longitudinale en levant les hypothèses d'indépendance entre les événements. Tous les travaux issus de l'approche transversale précédemment évoqués aboutissaient à la conclusion suivante: la mobilité est un phénomène complexe qui interagit avec d'autres phénomènes. Or, il est très difficile de saisir de façon satisfaisante ces interactions sans avoir recours à des outils et enquêtes spécifiques, l'idée directrice étant que les formes de mobilité sociale, géographique, familiale - "ne sont en réalité que les diverses facettes d'un processus unique de réaménagement de l'existence" (Grafmeyer, 1994). Alors que l'approche transversale induit peut-être un peu trop vite un raisonnement causal, du genre "le ménage change de logement parce que la taille de la famille s'est modifiée", l'approche biographique "permet de remplacer la notion de causalité par le concept plus

\footnotetext{
${ }^{3}$ L'analyse longitudinale, dont le développement en démographie est plus récent, consiste à suivre une population sur une durée de temps, plus ou moins longue, en étudiant un ensemble d'événements qui surviennent au cours de la période considérée.
} 
souple de dépendance locale qui permet de mieux approcher les interactions entre phénomènes" (Courgeau, 1988).

Dans ce type d'analyse, il s'agit de "restituer dans toute leur complexité les suites d'états et d'événements qui s'enchaînent au fil d'une même trajectoire de vie; la question de la mobilité se recompose autour de l'analyse des processus qui mettent en jeu tout à la fois des liens interpersonnels, des lieux géographiques et des statuts sociaux" (Grafmeyer). On signalera sans s'étendre davantage l'apport des historiens dans ce courant de recherche (Gribaudi, Pinol, Rosental, Lévy-Vroelant).

L'originalité de l'approche biographique réside dans le développement de nouvelles méthodes statistiques et la réalisation d'enquêtes permettant de les tester. Cette démarche et les collectes quantitatives ont été promues par des chercheurs spécialistes de la mobilité géographique (Courgeau, Lelièvre, 1989). Ces enquêtes permettent de resituer la mobilité par rapport aux événements qui jalonnent l'existence des individus. L'apparition de nouvelles méthodes mais aussi les progrès de l'informatique ont permis de dépasser l'approche purement individuelle en réalisant le passage de l'histoire d'un individu à celle d'un échantillon d'individus. Elles ont également permis le passage de l'analyse d'un parcours résidentiel à celle d'une biographie c'est-à-dire de plusieurs parcours en interaction: parcours résidentiel, familial, professionnel. Des mesures précises de ces interrelations sont désormais possibles.

Lanalyse des biographies va donc chercher à situer les changements qui interviennent dans un des domaines par rapport aux autres. Il s'agira de voir comment un événement familial, économique ou autre va modifier la probabilité d'arrivée des autres événements de l'existence (par exemple quelle est l'incidence de la naissance d'un enfant sur la mobilité résidentielle). L'hypothèse sous-jacente est que ces comportements ne sont pas déterminés, mais qu'ils se modifient au cours de l'existence sous l'effet des expériences personnelles. Ces méthodes ont connu un développement important, comme en témoigne le bilan de quatorze enquêtes biographiques réalisées au cours des vingt dernières années dans le monde (GRAB, 1999). En France, deux types d'interactions ont été particulièrement étudiés: entre mobilité professionnelle et mobilité géographique, entre vie familiale et mobilité résidentielle (voir les travaux de D. Courgeau).

Cette perspective biographique conduit à relativiser les découpages disciplinaires et nécessite d'autres registres de description et d'explication des faits de mobilité. Approches sociologiques, géographiques et démographiques convergent de plus en plus. La trajectoire résidentielle identifiée par une enquête longitudinale permet de comprendre le choix d'un logement, non comme un arbitrage à un moment donné, mais réellement comme un processus dynamique.

\section{Questions en Suspens, Thèmes de Débats}

Ces progrès dans la connaissance ne doivent pas masquer un certain nombre d'incertitudes, liées à la difficulté de mesurer les faits avec précision, et de saisir toute la complexité des éléments à prendre en considération pour pouvoir les interpréter. Les apports respectifs des différentes approches sont encore, on l'a vu, insuffisamment intégrés. Paradoxalement, la mobilité a donné lieu à un grand nombre d'études et de recherches, et donc à une accumulation considérable de connaissances, mais il subsiste des lacunes, et, lorsque l'on cherche à expliquer les évolutions passées ou à prévoir les changements à venir, les hypothèses et les résultats s'avèrent parfois fragiles, voire contradictoires.

\section{Problèmes de Mesure de la Mobilité}

La mesure de la mobilité résidentielle pose encore des problèmes, car les sources disponibles et les catégories statistiques utilisées ne sont pas toujours bien adaptées à l'étude d'un phénomène aussi complexe que la mobilité. Une estimation précise de données apparemment élémentaires, comme le nombre de personnes ayant changé de domicile au cours d'une année se heurte à de sérieuses difficultés.

La mesure de la décohabitation des jeunes adultes illustre bien ces difficultés.

Au cours des dernières années, on a observé en ce domaine un retard manifeste, les jeunes demeurant plus longtemps chez leurs parents que dans les années soixante-dix. Mais cette stabilité peut cacher une grande turbulence résidentielle, 
certains continuant à déclarer comme résidence principale celle de leurs parents, même si en réalité ils vivent la majeure partie du temps en dehors du domicile parental. Une même situation peut donc être enregistrée de façon différente selon la personne qui répond à l'enquête ou au recensement.

\section{Lacunes et Imperfections de LAppareil Statistique}

Une des difficultés majeures tient à la non-concordance des principales sources disponibles: d'un côté, le recensement, qui permet d'évaluer la mobilité individuelle, de l'autre, les Enquêtes Logement et le fichier EDF, qui renseignent sur la mobilité des ménages ${ }^{4}$. L'estimation des tendances sur une courte période impose de la prudence: en effet, les valeurs observées divergent selon les sources. Ainsi, l'analyse des résultats du dernier recensement fait apparaître une poursuite de la baisse de la mobilité, locale et lointaine. Mais, si on étudie les taux de déménagements à travers les Enquêtes Emplois ou les fichiers EDF, la mobilité semble remonter à partir du milieu des années quatre-vingt. Pour expliquer ces écarts, on peut avancer l'explication suivante: la mobilité des individus baisserait tandis que celle des ménages augmenterait. En effet, les ménages d'une ou deux personnes sont plus mobiles, alors que les "grands ménages" composés de couples avec deux ou trois enfants, et habitant en majorité une maison individuelle en milieu péri-urbain, sont beaucoup plus stables géographiquement.

\section{Problèmes d'Adéquation des Concepts}

Les recherches récentes montrent qu'il est difficile de saisir les interactions entre la mobilité résidentielle et $d$ 'autres phénomènes en utilisant les concepts statistiques de ménage et de lieu de résidence. En effet, l'évolution de la société est telle que certains repères deviennent plus flous. En tout cas, certaines catégories statistiques ne permettent plus de saisir les phénomènes dans leur complexité. Ainsi, le mariage d'autrefois entraînait généralement le déménagement d'au moins un des époux, tandis que, de nos jours, entre le départ du domicile des parents et la cohabitation, s'intercale souvent une période, parfois longue, pendant laquelle chacun des membres du futur couple a un logement.

\section{La Résidence}

L'exemple des jeunes montre toute la difficulté d'assigner un individu à un endroit fixe du territoire à un moment donné de son existence. De plus, grâce aux progrès de la technique, notamment dans les communications, un nouveau phénomène s'est considérablement développé, la "multilocalité". On connaît le cas des couples de fonctionnaires qui habitent séparément pour des raisons professionnelles, celui du professeur d'université qui passe une partie de la semaine dans une ville du domicile de sa famille, celui des retraités qui partagent leur temps entre deux résidences, des enfants de divorcés qui vivent chez leurs deux parents en alternance, ou encore celui des migrants étrangers qui investissent autant, voire davantage dans leur pays d'origine que dans le pays d'accueil.

D’une façon générale, on peut observer dans divers groupes sociaux une dissociation de plus en plus fréquente entre l'espace dans lequel l'individu s’investit réellement et l'espace du quotidien. On a là une question importante pour la compréhension des rapports entre les pratiques sociales et les formes urbaines. Plus précisément, il semble de moins en moins facile de cerner l'évolution des liens entre mobilité quotidienne et mobilité résidentielle à partir d'un seul lieu de référence déclaré officiellement "comme résidence principale". La multiplication des lieux d'habitat que l'on observe parmi les Français comme parmi les immigrés, conduit à se demander si la variable "résidence principale" n'est pas trop réductrice. Il apparaît donc utile de recourir à une notion plus large, comme celle "d'espace résidentiel" ou de "système résidentiel".

\section{Le Ménage}

Parallèlement à ce réexamen de la notion de lieu de résidence, les recherches démographiques et sociologiques ont conduit, dans les dernières années, à s'interroger sur l'utilisation du concept de ménage dans l'étude de la mobilité résidentielle.

\footnotetext{
${ }^{4}$ En France, le recensement indique seulement si un ménage se trouve dans un logement différent de celui qu'il occupait l'année du recensement précédent ; il sous-estime donc la mobilité résidentielle, omettant les changements de résidence qui ont pu avoir lieu dans l'intervalle (Baccaïni, 1999).
} 
Le concept de ménage relève de l'analyse transversale, car il désigne un groupe de co-résidents observés à un moment donné. Il est donc généralement impossible de suivre dans le temps sa mobilité résidentielle, car sa composition peut se transformer, par suite des naissances, décès, décohabitations, ruptures... C'est pourquoi les analyses longitudinales et biographiques portent sur les trajectoires - familiale, résidentielle et professionnelle - des individus, et sur leurs interactions. Toutefois, il ressort des recherches récentes que les caractéristiques individuelles ne sont pas les seuls indicateurs pertinents pour rendre compte de ces trajectoires. En effet, l'individu est rarement isolé: il est pris, le plus souvent, dans un ensemble de relations familiales, amicales et professionnelles qui influent sur ses choix de résidence. L'examen attentif des parcours résidentiels met en évidence le rôle de la famille étendue et des proches tout au long de l'existence, depuis la décohabitation jusqu'au choix de localisation au moment de la retraite, en passant par l'achat d'un logement ou par une entrée dans le parc social.

Ces analyses ont permis de prendre conscience des limites des concepts de famille et de ménage tels que l'INSEE les définit, car ils ne permettent pas d'appréhender le groupe ou les groupes qui accompagnent l'individu au cours de son histoire résidentielle, et à plus forte raison l'influence de tels groupes sur les trajectoires individuelles. En effet, les statistiques actuelles présentent, certes, un essai pour appréhender un tel groupe intermédiaire entre l'individu et la société. Mais, qu'il s'agisse du ménage ou de la famille, sa définition est ambiguë, la famille étant très souvent assimilée au groupe domestique ou considérée comme un sous-ensemble du ménage. Or, en réalité, on pourrait "inverser les termes, et se demander si ce n’est pas le ménage qui constitue un sous-ensemble de la famille" (Bonvalet, 1988). L'évolution de la société actuelle, avec la multiplication des familles mono-parentales et recomposées, l'apparition des semi-cohabitants, les recohabitations de jeunes chez leurs parents, fait que l'espace de la famille s'étend de plus en plus souvent au-delà des murs du logement et de son environnement immédiat. Certains auteurs ont donc estimé utile d'introduire la notion d'entourage dans l'analyse des mobilités résidentielles (Lelièvre et al., 1997). Un des défis de la recherche actuelle est d'observer les comportements de ces groupes intermédiaires et leur évolution dans la société contemporaine.

On peut donc se demander quel est l'agent pertinent, en matière de pratiques résidentielles. Quels concepts, et surtout quelles catégories statistiques doit-on et peut-on employer pour les appréhender avec précision? Ces problèmes se posent avec une acuité particulière dans l'étude des relations entre la mobilité résidentielle et la mobilité quotidienne. Classiquement, on considère que l'acteur dont il convient d'observer les pratiques pour analyser la mobilité quotidienne - et en tout cas le seul dont les déplacements peuvent faire sans trop de difficultés l'objet de comptages précis est l'individu - tandis que, dans la mobilité résidentielle, c’est le ménage qui est impliqué. Mais, outre les exemples ci-dessus, de nombreux travaux mettent en évidence la multiplicité des interactions entre les différents éléments pris en considération lorsqu'un ménage est confronté à un choix résidentiel: le plus souvent, sont en jeu une pluralité d'acteurs (parents et enfants, actifs et non-actifs, parfois des proches appartenant ou non à la famille...) et une pluralité de pratiques spatiales (trajets domicile - travail, déplacements liés aux études, aux loisirs, déplacements de week-end, déplacements saisonniers, etc.). La complexité de ces interactions ne peut que s'accroître, sous l'effet de l'allongement et de la diversification des cycles de vie individuels, ainsi que de la dissociation de plus en plus fréquente entre le ménage et la famille, ainsi qu'entre le ménage et le logement. C'est précisément, outre les migrations quotidiennes de travail, l'ensemble de ces déplacements et de ces interactions que l'on cherche à prendre en compte en recourant à des notions comme celles d'espace de vie ou de système résidentiel.

\section{Problèmes d'Interprétation des Liens entre la Mobilité Résidentielle et d'autres Types de Mouvements}

Les difficultés relatives à l'usage des outils statistiques traduisent, d'une certaine manière, la pluralité des facteurs qui influent sur la mobilité résidentielle. S'il est possible de tenir ensemble ces différentes facettes dans l'analyse d'un récit de vie, les difficultés s'avèrent considérables lorsqu'on passe à l'analyse des grands nombres. Sans doute des progrès ont-ils été réalisés, grâce 
au recours à des méthodes statistiques de plus en plus raffinées et surtout grâce à l'usage conjoint d'approches quantitatives et d'approches qualitatives: les rapprochements entre des outils tels que les analyses multidimensionnelles, les modèles logit, l'analyse textuelle, l'analyse biographique, etc., s'avèrent féconds. La mise en œuvre de recherches combinant analyse biographique et analyses multi-niveaux autorise, par exemple, à mieux prendre en compte les effets de contexte (Courgeau, Baccaïni, 1997).

\section{Questions sur les Rapports entre Demande Sociale et Analyse de la Mobilité}

Parce qu'elle met en jeu de nombreux processus économiques, sociaux, culturels, psychologiques, etc., la mobilité est considérée comme un bon analyseur du changement social, plus particulièrement dans le domaine de l'organisation de l'espace habité. De là viennent sans doute l'hétérogénéité des objectifs visés dans les recherches, et une part de leurs lacunes. Un réexamen critique de ces objectifs peut donc aider à mieux cerner les problèmes en suspens dans l'état actuel de la recherche.

L'étude de la mobilité a souvent servi d'outil pour la planification économique. C'est le cas, pour prendre un exemple éclairant, en ce qui concerne la connaissance des marchés de l'emploi. Si, dans certaines disciplines des sciences sociales (notamment l'histoire et la géographie, traditionnellement peu orientées vers l'action), l'étude des migrations a été longtemps conduite dans un simple souci de curiosité scientifique, depuis les années cinquante et soixante, un grand nombre de travaux répondent au besoin de prévoir et de maîtriser les fluctuations dans la répartition spatiale de la population active. Leurs orientations thématiques et méthodologiques sont largement soumises aux commandes qui financent la recherche. Aux préoccupations des responsables de l'aménagement répondent, notamment, les efforts de modélisation cités plus haut. Dans ces perspectives, l'approche quantitative et l'emploi de méthodes empruntées à l'analyse démographique ont été particulièrement encouragés. C'est en partie pour des raisons méthodologiques, mais pour une part en réponse à la demande des technocrates que s'est répandu l'usage du concept de mobilité. Celui-ci évoque une idée de progrès économique et social (de mobilité sociale, notamment) et on le préfère, peut-être inconsciemment, à celui de migration, qui fait davantage penser aux problèmes du déracinement (Brun, 1993).

La spécificité des thèmes suscités par la "demande sociale" a longtemps eu un revers: le caractère segmenté de la recherche. Si cette segmentation n'a pas totalement disparu, comme on l'a noté, la période actuelle se caractérise par la multiplication des efforts pour appréhender le phénomène de mobilité dans toutes ses dimensions conjointement. Les recherches qui visent à cerner les interactions entre la mobilité résidentielle et la mobilité quotidienne ou hebdomadaire sont particulièrement représentatives de cette tendance.

\section{Quelques Perspectives de la Recherche Actuelle}

On ne fera ici qu'énoncer quelques thèmes, parmi ceux qui font actuellement l'objet de travaux ou qui nous semblent pouvoir faire l'objet de projets de travail collectifs, en France, à l'intersection de l'étude de l'habitat et des mouvements migratoires.

Un axe de recherche appelé à un essor rapide est l'analyse des effets du progrès technologique, essentiellement dans les communications, sur les aspirations et les pratiques résidentielles. L’usage de nouvelles technologies (Internet, portable...) en facilitant le travail à distance pourrait dans quelques années modifier les arbitrages des ménages en terme de choix de localisation et de mobilité quotidienne. Il en va de même de l'augmentation de la vitesse des transports. La dilution de l'habitat autour des grandes métropoles en est la conséquence la plus éclatante. Encore faut-il tenir compte aussi de l'évolution du coût des déplacements, du confort des usagers, et peut-être aussi d'un élément de plus en plus prégnant: la fragilité de ces systèmes de migrations pendulaires, en cas de grèves, d'intempéries, etc. La mobilité dans les déplacements pendulaires n'a pas aboli toutes les contraintes d'accessibilité, et ne révolutionne que progressivement la mobilité résidentielle.

Ces recherches pourraient se focaliser notamment sur les choix effectués à certains moments du cycle de vie chez les jeunes au moment du passage à l'âge adulte, chez les familles monoparentales ou familles recomposées qui 
doivent gérer la multirésidence de leurs enfants, chez les personnes vieillissantes qui doivent faire face à une réduction de leur mobilité avec l'avancement en âge. Sur les points qui viennent d'être énoncés, il serait utile d'observer les relations entre mobilité quotidienne et mobilité résidentielle à l'aide d'approches non plus transversales, mais longitudinales, en étudiant comment, dans un panel donné de ménages, la façon de gérer ces arbitrages évolue, en fonction de facteurs externes et internes: changements dans la composition du ménage, dans la vie professionnelle de l'un ou l'autre conjoint, mais aussi transformations du contexte économique, social, urbain. Il pourrait enfin être intéressant d'étudier ces questions en ne se limitant plus à l'observation d'individus ou de ménages, mais en cherchant à cerner les pratiques d'un groupe de référence élargi, constitué de parents et d'amis proches ${ }^{5}$.

Les avancées de la recherche impliquent un effort de dépassement des systèmes de raisonnement binaires: pour comprendre les évolutions actuelles de la mobilité, il faut cesser d'opposer schématiquement l'individu au groupe, le quartier à la ville, le local au "global", la mobilité à l'enracinement... La poursuite des débats et collaborations interdisciplinaires s'impose donc. Les efforts déjà accomplis ont débouché, on l'a vu, sur d'importants acquis, et parmi eux, au premier chef sur l'acceptation, dans la plupart des disciplines, de la nécessité de dépasser l'opposition entre les approches microsociologiques et les approches fondées sur l'analyse de données agrégées. Reste un long travail à accomplir pour confronter systématiquement les explications à dominante psychologique, familiale, culturelle, technologique, économique...

Un autre plan sur lequel il apparaît souhaitable d'intensifier la recherche est celui de la prise en compte des effets exercés sur la mobilité résidentielle par les grands changements sociétaux.
En effet, on appréhende de manière assez floue l'impact de l'évolution économique sur la mobilité résidentielle. On objectera sans doute que, dans tous les travaux publiés, celle-ci est rapportée à de multiples "facteurs" ressortissant à l'économie: les revenus des ménages, le nombre d'actifs dans les ménages, leurs revenus, la localisation de leurs emplois, les loyers, les emprunts, etc. Mais, si des efforts d'analyse très précis sont engagés sur des questions comme celle de l'incidence des fluctuations monétaires et financières internationales sur les marchés immobiliers, au total, les liens entre la conjoncture économique générale et les tendances de la mobilité résidentielle demeurent assez mal connus.

Enfin, l'étude de la mobilité résidentielle dans ses relations avec les transformations des structures sociales est elle-même encore quelque peu embryonnaire sur bien des points ${ }^{6}$. Mais alors que l'on appréhende de mieux en mieux les liens entre mobilité et changements démographiques, que l'on mesure précisément les variations de la mobilité selon l'âge, le sexe, la composition de la famille, plus rares sont les analyses fouillées de ses variations selon l'appartenance à une classe sociale. On sait que l'accès à la mobilité pendulaire est socialement inégal, on sait soulever des questions pertinentes sur les inégalités d'accès aux formes "immatérielles" de mobilité. Mais des plages d'incertitude subsistent quant à leurs incidences en matière de mobilité résidentielle (et donc, à terme, en matière de formes de l'urbanisation...) et, surtout, à leurs rapports avec la diminution du temps de travail et la recomposition en cours des emplois et des qualifications. Ces zones d'ombre signifient-elles que l'on ne cerne pas très bien la redéfinition des hiérarchies sociales consécutive à la libération des échanges, à la globalisation et autres grands bouleversements de l'économie ? Tel sera pourtant un des défis de la recherche urbaine des prochaines années.

\footnotetext{
${ }^{5}$ L'étude des migrations à l'échelle des familles a donné lieu à des travaux historiques (Gribaudi, Rosental...). Mais, à l'exception d'un petit groupe de chercheurs de l'INED, peu de passerelles ont été établies jusqu'à présent entre ces approches et celles de la majorité des urbanistes, sociologues et géographes.

${ }^{6}$ Sans doute, là encore, faut-il se garder d'un jugement trop pessimiste, car des recherches fructueuses ont été faites sur certains aspects de ces mutations parmi les plus importants: par exemple sur la mobilité des travailleurs immigrés, des habitants du parc social, sur les S.D.F., sur la grande bourgeoisie...
} 


\section{Bibliographie}

ALONSO W., 1964, Location and Land Use, Cambridge, Harvard University Press.

ASCHER F., 1995, Métapolis ou l'avenir des villes, Paris, Odile Jacob.

BACCAÏNI B., 1999 - Recensement traditionnel et recensement rénové pour l'étude des migrations résidentielles, INSEE, Service Etudes et Diffusion.

BACCAÏNI B., COURGEAU D., DESPLANQUES G., 1993, "Les migrations internes en France de 1982 à 1990. Comparaison avec les périodes antérieures", Population, n. ${ }^{\circ} 6$.

BASSAND M., BRULHARDT M.-C., 1980, La mobilité spatiale, Saint Symphorien (Suisse), Editions Georgi.

BERGER M., 1999, "Mobilité résidentielle et navettes domicile-travail en Île-de-France", Espace, Populations, Sociétés, ${ }^{\circ}{ }^{\circ} 2$.

BLOSS T., GODARD F., 1990, "Décohabitation juvénile: stratégie juvénile et conjoncture de l'existence", in Bonvalet C., Fribourg A.-M., Stratégies résidentielles, INED, Plan Construction et Architecture, MELTM, Congrès et Colloques n. ${ }^{\circ} 2$.

BONVALET C. (ed.), 1994, Logement, mobilités et populations urbaines, Paris, Presses du CNRS, Cahier du PIR-Villes.

BONVALET C., Fribourg A.-M. (eds.), 1990, Stratégies résidentielles, Paris, Plan construction-INED, coll. Congrès et colloques, n. ${ }^{\circ} 2$.

BONVALET C, Brun J.,Segaud M (eds), 2000, Logement et habitat: bibliographie commentée- Paris, La documentation Française.

BRUN J., 1993, "La mobilité résidentielle et les sciences sociales. Transferts de concepts et questions de méthode", Annales de la recherche urbaine, $\mathrm{n} .{ }^{\circ} 59-60$.

CLARK W.A.V., ONAKA J.-L., 1983, "Life cycle and housing adjustment as explanations of residential mobility", Urban Studies, n. $^{\circ} 20$

COURGEAU D., 1976, "Mobilité géographique, nuptialité et fécondité”, Population, n. ${ }^{\circ}$ 4-5.

COURGEAU D., LELIEVRE E., 1989, Analyse démographique des biographies, Paris, INED.

CRIBIER F., KYCH A., 1992, "La migration de retraite des Parisiens. Une analyse de la propension au départ", Population, n. ${ }^{\circ} 3$.

DUREAU F. et al. (ed.), 2000, Métropoles en mouvement: une comparaison internationale, Paris, IRD, Anthropos, Collection Villes.
ESPACES et SOCIÉTÉS, 1988, Mobilités, Paris, L'Harmattan, n. ${ }^{\circ} 54-55$.

ESPACE, POPULATIONS, SOCIÉTÉS, 1999, Les mobilités, n. ${ }^{\circ} 2$.

GRAFMEYER Y., DANSEREAU F. (eds.), 1998, Trajectoires familiales et espace de vie en milieu urbain, Lyon, PUL.

GRIBAUDI M., 1982, "Stratégies migratoires et mobilité relative entre village et ville", Population, $n .{ }^{\circ} 6$.

GROUPE DE REFLEXION SUR L'APPROCHE BIOGRAPHIQUE, 1999, Biographies d'enquêtes: bilan de 14 collectes biographiques, Paris, INED, PUF, Méthodes et Savoirs, n. ${ }^{\circ} 3$.

LELIEVRE E., LEVY-VROELANT C., 1992, La Ville en mouvement: habitat et habitants, Paris, L'Harmattan.

LELIEVRE E., 1999, "Collecter des données de mobilité: des histoires migratoires aux biographies d'entourage" Espace, Populations, Sociétés, Les mobilités, $\mathrm{n}^{\circ} 2$.

LEVY J.-P., 1993, "L'habitat influe-t-il sur la mobilité ? La mesure de la mobilité résidentielle en France", Les Annales de la Recherche Urbaine, n. ${ }^{\circ} 59-60$.

LEVY J.-P., 1998, "Dynamique du peuplement résidentiel", Société contemporaine, $\mathrm{n}^{\circ} 29$.

LEVY J.-P., DUREAU F. (ed), 2002, L'accès à la ville. Les mobilités spatiales en question. Paris, Antrhopos (coll.Villes).

PENTEL A., JAYET H., 1995, Les facteurs économiques de la mobilité résidentielle. Une enquête préliminaire, Rapport au CNRS-PIR-Villes, CESURE.

RHEIN C., 1990, "Mobilité résidentielle et dynamique urbaine", Revue de Géographie de Lyon, vol. 65, n. 3 .

ROSEN S., 1974, "Hedonic Prices and Implicit Markets: Product Differentiation in Pure Competition", Journal of Political Economy, 82, 1.

ROSSI P.-H., 1955, Why families move: a study in the Social Psychology of Urban Résidential Mobility, Glencoe, The Free Press.

SEGAUD M., BONVALET C., BRUN J. (dir.), 1998, Le logement et l'Habitat, l'état des savoirs, Paris, La Découverte.

SEGAUD M., BRUN J, DRIANT J. C. (dir.), 2003, Dictionnaire de l'Habitat et du logement, Paris, Armand Colin.

TARRIUS A., 1992, L'Europe des fourmis, Paris, L'Harmattan.

SIMMONS J., 1968, "Changing Residence in the City. A review of intraurbain mobility", Geographical Review, vol. LVIII, n. ${ }^{\circ} 4$. 